\title{
Experimental Investigation of Magnetic Self-Assembly for Swallowable Modular Robots
}

\author{
Zoltán Nagy, Raymond Oung, Jake J. Abbott, and Bradley J. Nelson
}

\begin{abstract}
There is a clear trend toward the miniaturization of medical devices for minimally invasive medical procedures, ranging from diagnosis and targeted drug delivery to complex surgical interventions. Current research focuses on increasing the functionality of commercially successful capsule endoscope technology by developing active locomotion and telemetry. The size of such a capsule must not be larger than what a person can swallow without difficulty. One approach to increase functionality while still working within this size constraint is to build a modular robotic system in which the modules are swallowed one at a time, and the final assembly is performed inside the gastrointestinal (GI) tract. This paper addresses a fundamental challenge that must be be met for the success of such swallowable modular robots-their self-assembly. We propose to use magnets in a specific configuration on the mating faces of the modules. Our results show that high success rates can be achieved and snake-type robots can be self-assembled with compliant magnetic joints allowing them to adapt to highly irregular paths, such as the small intestine.
\end{abstract}

\section{INTRODUCTION}

There is a clear trend toward the miniaturization of medical devices for minimally invasive medical procedures ranging from diagnosis and targeted drug delivery to complex surgical interventions. One commercially successful device, introduced in 2001, is the M2A capsule endoscope [1], a camera pill with a length of $26 \mathrm{~mm}$ and diameter of $11 \mathrm{~mm}$ that is swallowed and delivers images as it moves through the gastrointestinal (GI) tract. Its essentially non-invasive nature allows for less painful diagnosis than traditional endoscopy, as well as imaging of the small intestine, which is not possible with conventional endoscopes. However, its functionality is limited to passive sliding through the GI tract and imaging at random orientations. The Sayaka capsule endoscope was commercialized in 2005 , with the ability to rotate along its axis while imaging the walls of the intestine [2]. For the state of the art in capsule endoscopy, see [3].

Current research focuses on increasing the functionality of capsule endoscopes by developing active locomotion [4][6] and remote monitoring of $\mathrm{pH}$, temperature, conductivity, and oxygen concentration [7]. However, the size of such a device must not be larger than what a person can swallow without difficulty. An international clinical study on complications and limitations of capsule endoscopy ( $>700$ cases) concluded that in $16 \%$ of the cases, problems occurred due to difficulty or inability of the capsule to pass through the GI

This work was supported by the European Commission in the framework of the 6FP NEST Adventure Project ARES.

The authors are with the Institute of Robotics and Intelligent Systems, ETH Zurich, Switzerland. Z. Nagy is the corresponding author (nagyz@ethz.ch).
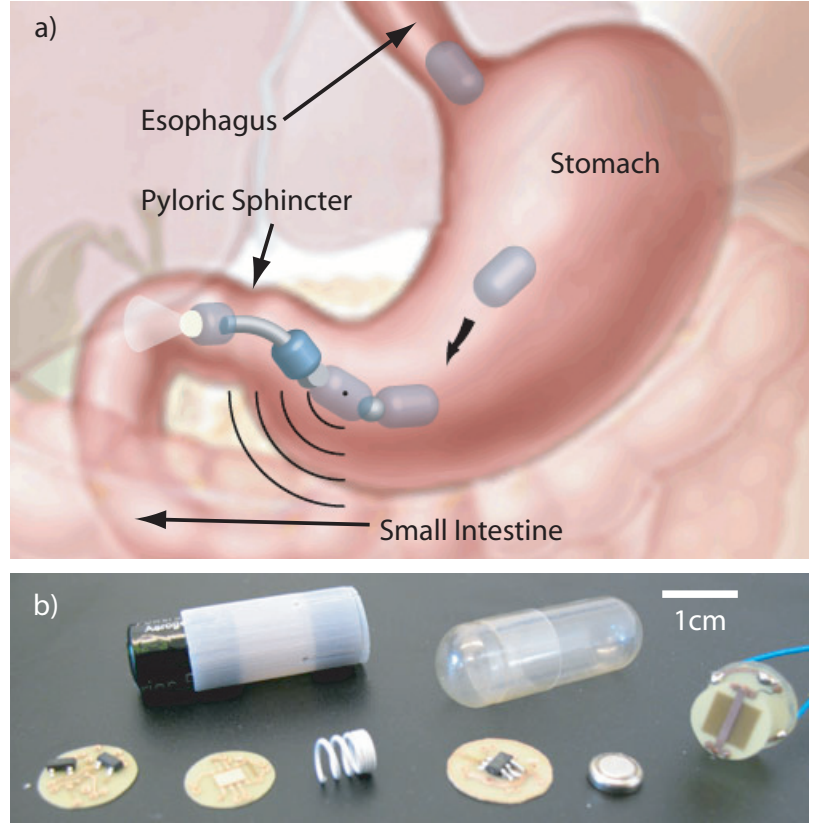

Fig. 1. a) Artistic concept of a snake-type swallowable modular robot (SMR) assembled in the stomach and moving into the intestine. b) Components of modules of an SMR currently developed in our group, in comparison to a commercial pharmaceutical capsule.

tract [8]. Therefore, the aforementioned size of the capsule represents a rigid upper limit for swallowable devices.

One approach to overcome this size constraint is to build a modular robotic system in which the modules are swallowed one at a time and the final assembly is performed inside the GI tract. This allows the size and the complexity of the final robot to be significantly greater than that of its individual modules. If the modules are designed to be heterogeneous, each having a specific dedicated function, the difficulty in fabrication and packaging will be reduced, and relatively large devices could be included in the robotic system. As an example, consider a snake-type robot with heterogeneous modules that assembles in the stomach and then moves through the intestine, as shown in Fig. 1. At its front end, sensors detect pathologies that are communicated to the surgeon, who decides on the actions to take. A module designed for a specific task executes the commands once the robot has been positioned accurately by an actuation module. As mentioned above, sensors and actuators for pill-sized devices are currently being developed, and each sensor/actuator could constitute an individual module. 


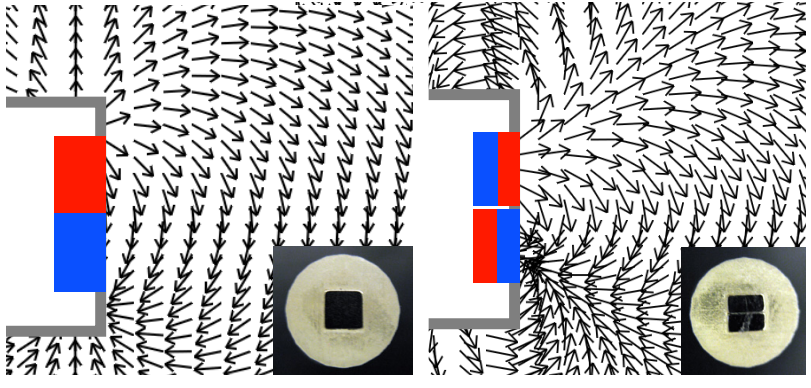

Fig. 2. Field plots of the MASH-1 (left) and MASH-2 (right) configurations. The insets show actual prototype modules used in the experiment.

After a procedure, the robot may remain in its snake-type shape while moving through the intestine, or reconfigure into another shape, or disassemble completely. Regardless, the fundamental challenge that must be met for the success of any such swallowable modular robots (SMR) is their selfassembly. Due to their long-range passive interaction, the use of magnets as the driving mechanism of self-assembly seems self-evident: if magnets are placed on the mating faces, the magnetic force will attract the different modules toward each other and the magnetic torque will orient them. In addition, magnets provide a reversible connection allowing for disconnection and reconfiguration. However, it is not evident what magnetic configuration (i.e., number and orientation of the magnets) to use in order to achieve the highest probability for a successful connection. It is desirable that each module is able to connect to any other module to increase the number of possible topologies of the robot, and that the mating faces connect in a unique orientation to enable a priori design of the possible kinematic configurations without the need for additional position/orientation sensors.

Nagy et al. [9] recently introduced the concept of the MAgnetic Self-aligning Hermaphroditic (MASH) mating face and argued that, for a unique connection, the mating faces should present exactly two opposite magnetic poles. The two conceivable realizations, as shown in Fig. 2, are with one magnet oriented such that the connection takes place along an axis perpendicular to the magnetization of the magnet (MASH-1), and with two distinct magnets (MASH2). Experiments with upscaled models of single MASH-1 mating faces under quasi-static conditions, as well as magnetic field analysis, suggested that the MASH-1 configuration is useful for self-assembly and alignment. However, due to the dynamic and random nature of the assembly process, experiments under more realistic conditions are necessary to investigate which configuration provides superior selfassembly.

During self-assembly, the modules move such that the free energy of the system is minimized. Due to the large number of forces involved (gravity, magnetic force/torque between the modules, fluid drag on the modules, the friction between touching modules, and the friction between the modules and the GI-tract wall), as well as the randomness of initial conditions, multiple local energy minima are present.
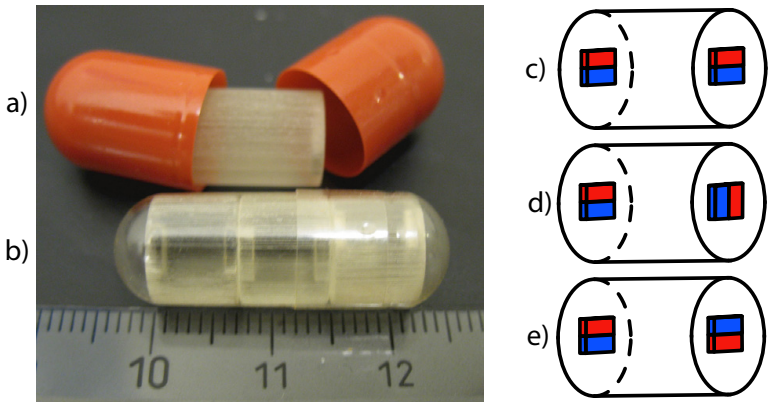

Fig. 3. a) A long module in a DBAAA-sized module. b) Three short modules in a 000 sized capsule. c-e) parallel, perpendicular, and antiparallel orientation. Only MASH-1 modules are shown for simplicity.

Thus, in addition to the desired successfully connected state, other states are possible ranging from misaligned assembly to no interaction at all. No numerical tools are available to capture all the above physical effects in a single simulation. Therefore, in this paper we experimentally investigate selfassembly using modules with MASH mating faces and find that high success rates can be achieved.

Our results also extend to the area of search-and-rescue and inspection robots where the actual working or assembly area is larger than the access point. Modular robots nominally designed for these applications often use magnets as connectors [10]. However, the mating faces are typically brought together prealigned by other actuators in order to avoid misalignments - a connection method that is appropriate for reconfiguration of modular robots, but is not sufficient for the type of initial self-assembly that is the goal of this work.

There is a body of recent research dealing with magnetic self-assembly of cm-sized and smaller objects. Approaches include the incorporation of soft and hard magnets on planar sheets that can fold into three-dimensional structures [11], [12], and the use of magnet arrays on the parts to be assembled that are brought close together, letting the magnets finish the alignment process [13]. However, there is no prior work that deals with magnetic self-assembly of free-floating objects.

\section{Prototype Modules}

We have developed a set of simple modules to abstract the problem of self-assembly and study it experimentally. We consider cylindrical modules with length $7 \mathrm{~mm}$ (short) and $14 \mathrm{~mm}$ (long) and diameter $9 \mathrm{~mm}$. These sizes are chosen such that three small modules fit into a ConiSnap ${ }^{\circledR}$ [14] capsule of size 000 (length $26.1 \mathrm{~mm}$, outer diameter $9.5 \mathrm{~mm}$ ), and the long modules having twice the length of the short ones (see Fig. 3a)-b)). In addition to size 000, the DBAAAsized capsules (length $23 \mathrm{~mm}$, outer diameter $11 \mathrm{~mm}$ ) are also conceivable as carriers for robot modules. As the two capsules are the largest commercially available, they represent an approximate upper limit of such modules. By using modules of this size, we expect no significant scaling effects, and our findings translate directly to a final SMR. 


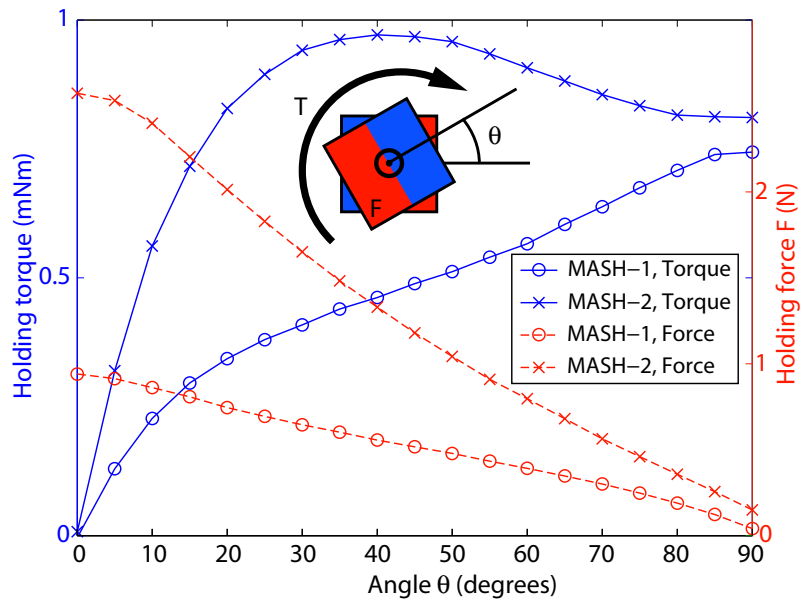

Fig. 4. Holding torque $T$ and force $F$ as a function of the orientation of the modules computed by FEM. In the inset, $F$ is perpendicular to the drawing plane and only MASH-1 type magnets are shown for simplicity. For the FEM, the module faces are separated by $0.1 \mathrm{~mm}$ to avoid numerical instabilities.

The modules are fabricated out of an acrylic-based photopolymer by rapid prototyping, and permanent magnets are glued into the designated openings at both ends of the modules. We explore three different possible arrangements of the magnets on a given module, as shown in Fig. 3c)e): parallel, perpendicular, and antiparallel. The orientation is not significant for the short range interaction between two modules (for the sizes and magnets investigated in this work), because the shape of the magnetic field is locally dominated by a single MASH face. In the long range, on the other hand, the field is shaped by both MASH faces. Therefore, an influence of the orientation is expected.

For the MASH-1 configuration, one $3 \mathrm{~mm} \times 3 \mathrm{~mm} \times 1 \mathrm{~mm}$ magnet is used on each face, whereas for the MASH-2 configuration, two $3 \mathrm{~mm} \times 1.5 \mathrm{~mm} \times 1 \mathrm{~mm}$ magnets are used. The result is a $3 \mathrm{~mm} \times 3 \mathrm{~mm}$ magnetic face with a depth of $1 \mathrm{~mm}$ for both MASH types (see insets in Fig. 2). The magnets are $\mathrm{NdFeB}$ magnets with a remanence of $1.4 \mathrm{~T}$.

\section{STATIC INTERACTION ANALYSIS}

We analyze two static situations for their correlation with the statistical experimental results: short- and long-range interaction. In the short range, when the two mating MASH faces are closer than four times the size of the magnet $(<12 \mathrm{~mm}$ in our case), we investigate the holding force and the restoring torque. Since the size of the magnets and their shape considerably influences the interaction, finite element (FE) analysis is performed (Maxwell 3D v12). Starting from the connected and aligned configuration, one module is rotated by the angle $\theta$ about the $z$ axis and the force $F(\theta)$ and the torque $T(\theta)$ are computed.

The results are shown in Fig. 4. It was observed that neither the module size nor the arrangement of the magnets has significant influence, which is reasonable as the two close MASH faces dominate the interaction. Therefore only

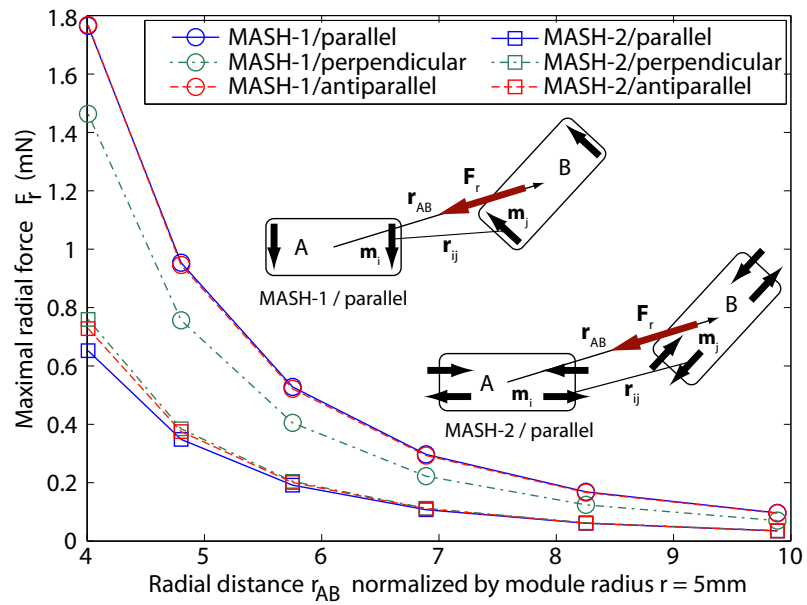

Fig. 5. The long range interaction model predicts up to three times higher attractive forces for the MASH-1 modules.

the results for short modules with parallel orientation are shown. We observe that the restoring torque, as well as the holding force, for the MASH-2 configuration is up to twice as large as the torque and force for the MASH-1 configurations. Figure 4 also gives an indication for a possible disconnection mechanism: in the connected state $\left(\theta=0^{\circ}\right)$ the restoring torque is zero and the holding force is maximal. Thus, initial twisting of the modules is easier than directly pulling them apart, and as $\theta$ is increased, the force needed for disconnection is reduced.

In the long range, when the MASH faces are distant by more than four times the size of the magnet, the magnet can be modeled as a point dipole located at the magnet's center. Consider two modules $A$ and $B$, where the center of module $B$ is described relative to module $A$ by $\mathbf{r}_{A B}$. Let $\mathbf{F}_{A B}$ denote the total force acting on module $B$ due to module $A$. Then, $\mathbf{F}_{A B}$ is the sum of the forces acting on the dipoles of $B$ (see insets Fig. 5):

$$
\mathbf{F}_{A B}=\sum_{i=1}^{\alpha} \sum_{j=1}^{\alpha} \mathbf{F}\left(\mathbf{m}_{i}, \mathbf{m}_{j}\right) \quad \text { with } \alpha=\left\{\begin{array}{l}
2, \text { if MASH-1 } \\
4, \text { if MASH-2 }
\end{array}\right.
$$

where $\mathbf{F}\left(\mathbf{m}_{i}, \mathbf{m}_{j}\right)$ is the force acting on dipole $\mathbf{m}_{j}$ of module $B$ due to dipole $\mathbf{m}_{i}$ of module $A$ and is given by [15]

$$
\begin{aligned}
\mathbf{F}\left(\mathbf{m}_{i}, \mathbf{m}_{j}\right)= & \frac{3 \mu_{0}}{4 \pi\left|\mathbf{r}_{i j}\right|^{5}}\left[\left(\mathbf{m}_{i} \cdot \mathbf{m}_{j}\right) \mathbf{r}_{i j}\right. \\
& +\left(\mathbf{m}_{i} \cdot \mathbf{r}_{i j}\right) \mathbf{m}_{j}+\left(\mathbf{m}_{j} \cdot \mathbf{r}_{i j}\right) \mathbf{m}_{i} \\
& \left.-5 \frac{\left(\mathbf{m}_{i} \cdot \mathbf{r}_{i j}\right)\left(\mathbf{m}_{j} \cdot \mathbf{r}_{i j}\right)}{\left|\mathbf{r}_{i j}\right|^{2}} \mathbf{r}_{i j}\right]
\end{aligned}
$$

where $\mathbf{r}_{i j}$ is the vector pointing from dipole $\mathbf{m}_{i}$ to dipole $\mathbf{m}_{j}, \mathbf{m}=v \mathbf{M}$ is the dipole moment (in $\mathrm{Am}^{2}$ ), with $\mathbf{M}$ the mean magnetization (in $\mathrm{A} / \mathrm{m}$ ) and $v$ the volume of the magnet (in $\mathrm{m}^{3}$ ), and $\mu_{0}=4 \pi \times 10^{7} \mathrm{Tm} / \mathrm{A}$ the permeability of free space [15]. For the assembly we are only interested in the component of $\mathbf{F}_{A B}$ along the direction $\hat{\mathbf{r}}_{A B}=\mathbf{r}_{A B} /\left|\mathbf{r}_{A B}\right|$ : $F_{r}=\hat{\mathbf{r}}_{A B} \cdot \mathbf{F}_{A B}$. 
MATLAB was used to analyze the interaction forces based on $F_{r}$ as follows: module $A$ is placed at the origin in a known pose. Then, for a given radial distance $\left|\mathbf{r}_{A B}\right|, 1000$ random vectors $\mathbf{P} \in[0,2 \pi] \times[0,2 \pi] \times \mathbb{R}^{3}$ are generated where the first two elements are the remaining spherical coordinates of module $B$ with respect to $A$, and the final three numbers parametrize the orientation of module $B$. Now, for each $\mathbf{P}, F_{r}$ is computed for each of the three investigated parameters (MASH type, module size, magnet orientation) and the statistical distribution of $F_{r}$ is observed.

The mean value of $F_{r}$ is zero, as repulsive and attractive forces are equally likely, so the standard deviation $\sigma$ is used as indicator of the range of possible forces. Figure 5 shows the results plotting $+3 \sigma$ versus $\left|\mathbf{r}_{\mathbf{A B}}\right| / r$, where $r=$ $5 \mathrm{~mm}$ is the radius of the modules. Since the dipole-dipole distance on one module becomes negligible for large $\left|\mathbf{r}_{A B}\right|$, no significant difference between short and long modules is observed, and only the data for short modules is shown. It can be seen that the MASH-1 configurations provide a force that is roughly twice as large as the force provided by the MASH-2 configurations. Thus, MASH-1 configurations are favorable for attracting modules towards each other. It can also be observed that for the MASH-2 configurations, every orientation provides a similar force, whereas for the MASH1 configuration the perpendicular orientation provides an approximately $20 \%$ smaller force than the parallel and the antiparallel configuration.

Considering $F_{r}$ only as an attractive force is motivated by our experiments (see Section IV) in which we observed that repulsion is an unstable state due to magnetic torque, and the modules tend to rotate into the stable attractive state.

\section{EXPERIMENTAl SELF-Assembly RESUlts}

The self-assembly performance for a given design is investigated by the following experiment: first, from a given location, one module is dropped into a recepticle filled with a cup of water $(\approx 300 \mathrm{~mL})$. The water simulates the water that is consumed by the patient when swallowing the pills. After having waited for the first module to settle, the second module is dropped from the same position but at a random orientation. Two different recepticles are used: a tapered bowl (height $75 \mathrm{~mm}$, upper diameter $120 \mathrm{~mm}$, lower diameter $50 \mathrm{~mm}$ ), as well as an anatomically correct model of a dilated stomach (volume 1.4L) milled out of acrylic glass (Fig 6). Using the bowl allows us to draw general conclusions and correlate our findings to the presented models. On the other hand, the stomach model has a prefered direction and a curved bottom, and we can study the self-assembly process at scale.

For each configuration, 50 drops are performed and the percentage of successful connections is recorded, as are the different misalignments that occurred. In total we observed seven different states: the successful connection (S), five stable misalignments (1-5), and a no-interaction state (X) (see Fig. 7a). Figure 7b) shows the experimental results. For the bowl, we observed up to $62 \%$ and $30 \%$ success rate for the long and short MASH-1 modules respectively,

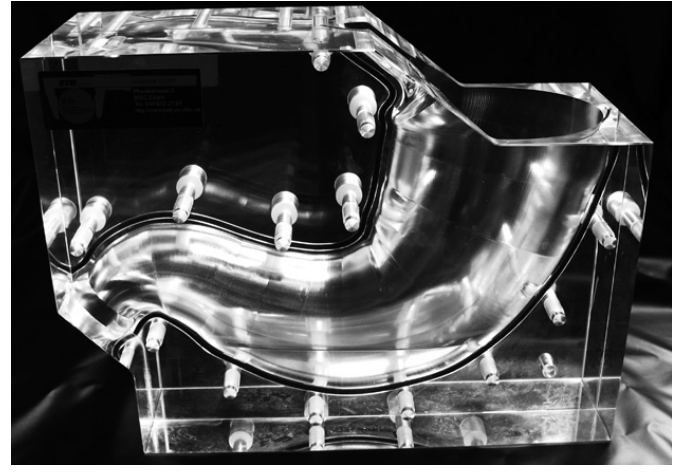

Fig. 6. Stomach model used for the self-assembly experiments

and $10 \%$ and $7 \%$ for the long and short MASH-2 modules. Clearly, for both the short and the long modules, the MASH1 configuration provides a much higher success rate than the MASH-2 modules. This is in agreement with the longrange model in that a higher attractive force correlates with successful self-assembly. Although MASH-2 exhibits a higher holding force once assembled, in the long range the fields of the two magnets of a given MASH-2 mating face effectively cancel; we observed up to $68 \%$ of the noconnection state for the long MASH-2 modules. Overall, the results are comparable for the stomach model, with minor changes for the states that favor/penalize the size/shape of the stomach.

As for the size of the modules, we find that large modules tend to assemble better than short ones. As the static models neglect the size difference, this is likely due to dynamic and friction effects. Since the difference is significant $(62 \%$ $(60 \%)^{1}$ for long MASH-1 vs. $30 \%$ (36\%) for short MASH1 ), further investigation is required to deepen our understanding of the self-assembly process.

The highest overall assembly success rate in the bowl, $62 \%$, occurred with a long module with MASH-1 faces arranged perpendicularly. However, the analytical model predicted a somewhat lower force for the perpendicular configuration. Clearly, a higher force is not necessarily the best indicator for the self-assembly performance.

Although repulsive and attractive forces are equally likely, success rates $>50 \%$ indicate that force is not the sole contributor for successful self-assembly. In fact, the magnetic torque also has a long-range effect by orienting a magnet along the field direction at a given location. In our experiments, we observed that this torque has a stronger effect than the repulsive force, in that the modules prefered to rotate into an attractive orientation rather than being repelled. The magnetic torque depends on the magnetic field strength in a given location [15]. As the magnets on a MASH-2 face cancel their respective field, the torque that can be exerted from such a face is virtually eliminated. On the contrary, the MASH-1 faces exhibit comparatively large torques and

\footnotetext{
${ }^{1}$ In the following, the numbers in the brackets represent the results for the stomach model
} 

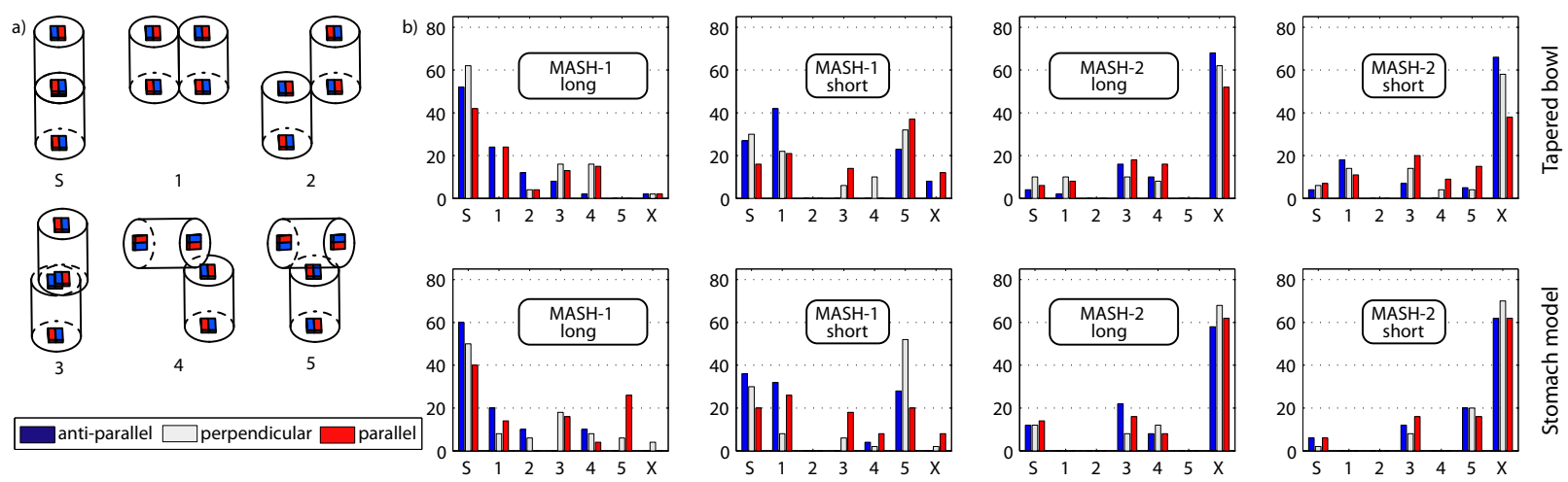

Fig. 7. a) Observed stable states during the drop experiments: successful connection (S) and five misalignments (1-5). Only antiparallel MASH-1 modules are shown, the states for the other modules are comparable. b) Results of the drop experiments. The percentage occurrence of the different states is shown. The no-interaction state is indicated by X. Clearly, for both the short and the long modules, the MASH-1 configuration provides a much higher success rate than the MASH-2 modules. In addition, very few no-interaction states are observed with MASH-1.

are able to orient a module such that the long-range force attracts the modules toward each other. This behavior is also influenced by the relationship between mass and rotational inertia, and needs to be taken into account for an actual module design.

Depending on the actual task of the robot, some of the misaligned states can be considered as successful connections. For example, state 3 is kinematically similar to the (most stable) success state. In addition, states 2,4 and 5 have been observed to be comparatively unstable and little excitation caused them to reorient into state 3 or the success state. Such an excitation can be provided by applied magnetic fields, the movement of the stomach, or the pyloric sphincter. Under these considerations the self-assembly success rate would be greatly improved.

The FE analysis showed that holding force and torque of the MASH-2 modules are higher than for the MASH-1 modules. Therefore, for an assembled structure where high intermodule forces are required, the MASH-2 configuration is favorable. On the other hand, it is also possible to increase the holding force by increasing the size of the MASH-1 magnets, which also favors the self-assembly performance. We performed the drop experiment with long MASH-1 modules with $4 \mathrm{~mm} \times 4 \mathrm{~mm} \times 2 \mathrm{~mm}$ sized magnets oriented perpendicularly. For the bowl, this increased the success rate to $74 \%$. Furthermore, states 3 and 4 were reduced to $8 \%$ and $10 \%$ respectively, and the no-interaction state was not observed at all. As in the previous experiment with the smaller magnets, states 1 and 5 did not occur. However state 2 occured twice as often as before $(8 \%)$. For the stomach model, the larger magnets resulted in the same success rate of $74 \%$, eliminated states 3 and 5 and reduced the states 1 and 2 to both $4 \%$. Only state 4 was increased to $18 \%$.

Clearly, these experiments simplify the real process, in that the stomach wall properties and the peristaltic motion of the stomach are neglected. However, the stomach wall is rather slippery, therefore minimal friction effects are expected. Furthermore, the motion of the stomach adds external energy to the whole self-assembly system, allowing it to potentially reconfigure from an undesirable into a more stable desirable configuration. To conclude, even though the real stomach properties are neglected, the obtained statistical results help to build intuition on magnetic self-assembly.

\section{Compliant Magnetic Joints}

It is desirable that the assembled robot can adapt itself passively to the unstructured topology of the GI tract. For this, specific modules could be designed with the sole task to deform easily. Alternatively, ferromagnetic joints can be attached to the modules as shown in Fig. 8a), where a cylindrical magnet (length $10 \mathrm{~mm}$, diameter $4 \mathrm{~mm}$, remanence $1.3 \mathrm{~T}$ with diametral magnetization) is assembled to a long MASH1 module with perpendicular orientation. The cylinder allows for an unactuated rotational degree of freedom between two modules, since the modules can slide with respect to each other on the surface of the cylinder. However, due to the magnetization of the cylinder, it also exerts a magnetic restoring torque on the connected magnets and thus orients the modules into the most stable state.

For self-assembly, preassembled cylindrical joints are greatly beneficial. To demonstrate this, we performed another drop experiment in which the first module was the one shown in Fig. 8a) and the second one was the regular module without joints. We observed only the three stable states shown in Fig. 8b)-d), where the first two each occurred in $45 \%(50 \%)$ of the experiments, the last one $10 \%(0 \%)$, and the no-interaction state was never observed. Note the difference between the symmetrical state in Fig. 8b), and the asymmetrical state in Fig. 8c). In both cases, however, a restoring torque returns the modules into the depicted stable state.

We can use such joints together with a specific assembly strategy to self-assemble snake-type robots as shown in Fig. 9. It assembled after dropping a module with a single magnet, then a module with two perpendicular joints, and finally another module with a single magnet. Repeating this procedure can result in a robot that can conform to highly irregular paths, such as the small intestine. 


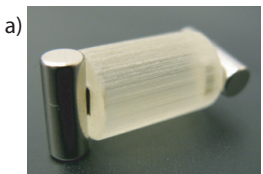

d)

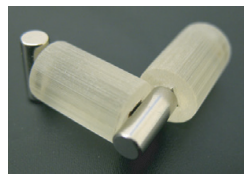

b)
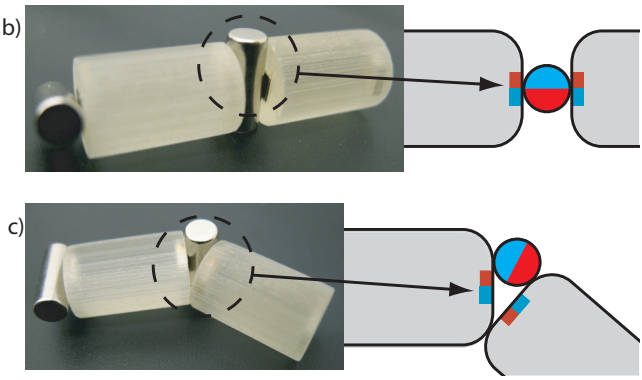

Fig. 8. a) Cylindrical magnets attached to a long MASH-1 module with perpendicular configuration provide a degree of freedom between two assembled modules. b)-d) Stable states that have been observed in the experiments. The sketches are cross-sections drawn to scale.

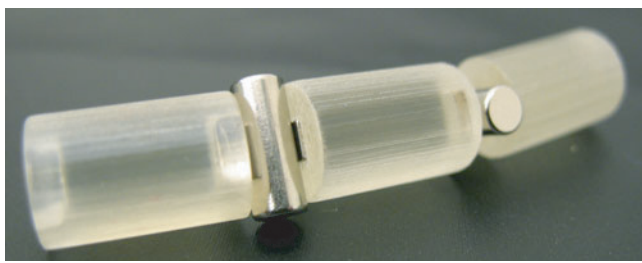

Fig. 9. Self-assembled 2-DOF snake-type modular robot

\section{CONCLUSIONS}

Our experiments show that the MASH-1 modules are able to successfully achieve self-assembly from random initial conditions. This is in agreement with the long-range model predicting higher attractive forces for MASH-1 modules. In addition, the low occurrence of the no-interaction state $(\leq 2 \%)$ makes the MASH-1 modules particularly interesting, as in virtually any case, the modules will self-assemble. On the other hand, MASH-2 modules are interesting if hermaphroditic mating faces with high intermodular forces are required, and self-assembly is not a critical issue.

We also find that although MASH-2 faces generate the greatest holding force and torque, and the parallel and antiparallel MASH-1 modules exhibit the largest long-range attractive force, it is the perpendicular MASH-1 modules that demonstrated the best ability to self-assemble. This is due to the fact that none of the static models are able to predict misalignments. Therefore, experiments, or at least accurate dynamic multiphysics simulations, are necessary to investigate magnetic self-assembly of $\mathrm{cm}$-sized objects.

We demonstrated self-assembly of snake-type robots by preattaching magnetic cylindrical joints to the modules. These joints can allow the robot to adapt itself to the GI tract as it moves through it while still maintaining intermodule connection.

To further increase the probability of correct alignment for MASH-1, the following measures are proposed. First, the magnet size should be optimized with respect to the actual geometry and inertia of the modules. Magnets that are too small do not guarantee sufficiently high interaction forces, whereas large magnets might induce undesirable yet stable misalignments. Shaping the geometry of the mating face, while still maintaining its hermaphroditic nature, changes its frictional behavior and will allow the misaligned states to reorient into the successful connected state. Finally, the magnetic field of the MASH-1 faces can be reshaped by introducing soft-magnetic shielding material near the magnet to guide its flux, with the potential to reduce the occurrence of misalignments.

\section{ACKNOWLEDGMENTS}

The authors would like to thank Kanako Harada and Arianna Menciassi from CRIM, Scuola Superiore Sant'Anna, Pisa, for fruitful discussions, and Sara Condino from the same institution for the CAD data of the stomach model.

\section{REFERENCES}

[1] (2008). [Online]. Available: http://www.givenimaging.com

[2] (2008). [Online]. Available: http://www.rfsystemlab.com/

[3] A. Moglia, A. Menciassi, M. O. Schurr, and P. Dario, "Wireless capsule endoscopy: from diagnostic devices to multipurpose robotic systems," Biomedical Microdevices, vol. 9, no. 2, pp. 235-243, 2007.

[4] B. Kim, S. Lee, J. H. Park, and J.-O. Park, "Design and fabrication of a locomotive mechanism for capsule-type endoscopes using shape memory alloys (SMAs)," IEEE/ASME Trans. on Mechatronics, vol. 10, no. 1 , pp. 77-86, 2005 .

[5] C. Stefanini, A. Menciassi, and P. Dario, "Modeling and experiments on a legged microrobot locomoting in a tubular, compliant and slippery environment," Int. J. Robotics Research, vol. 25, no. 5-6, pp. 551-560, 2006.

[6] M. E. Karagozler, M. E. Karagozler, E. Cheung, J. Kwon, and M. Sitti, "Miniature endoscopic capsule robot using biomimetic micropatterned adhesives," in IEEE/RAS-EMBS Int. Conf. on Biomedical Robotics and Biomechatronics, 2006, pp. 105-111.

[7] E. A. Johannessen et al., "Implementation of multichannel sensors for remote biomedical measurements in a microsystems format," IEEE Trans. Biomed. Eng., vol. 51, no. 3, pp. 525-535, 2004.

[8] E. Rondonotti, J. M. Herrerias, M. Pennazio, A. Caunedo, M. Mascarenhas-Saraiva, and R. de Franchis, "Complications, limitations, and failures of capsule endoscopy: a review of 733 cases," Gastrointestinal Endoscopy, vol. 62, no. 5, pp. 712-716, 2005

[9] Z. Nagy, J. J. Abbott, and B. Nelson, "The magnetic self-aligning hermaphroditic connector: A scalable approach for modular microrobots," in IEEE/ASME Int. Conf. Advanced Intelligent Mechatronics, 2007.

[10] S. Murata, E. Yoshida, A. Kamimura, H. Kurokawa, K. Tomita, and S. Kokaji, "M-tran: self-reconfigurable modular robotic system," IEEE/ASME Trans. Mechatronics, vol. 7, pp. 431-441, 2002.

[11] M. Boncheva et al., "Magnetic self-assembly of three-dimensional surfaces from planar sheets," Proc. of the National Academy of Sciences of the U.S.A., vol. 102, no. 11, pp. 3924-3929, 2005.

[12] E. Shechter, A. Arumbakkam, P. Lamoureux, X. Tang, M. Shima, and S. Akella, "Towards batch fabrication and assembly of 3D microstructures: A sequential assembly planner with new hard magnet configuration," in Proc. IEEE/RSJ Int. Conf. Intelligent Robots and Systems, 2007, pp. 584-589.

[13] S. B. Shetye, J. S. Agashe, and D. P. Arnold, "Investigation of microscale magnetic forces for magnet array self-assembly," IEEE Trans. Magn., vol. 43, no. 6, pp. 2713-2715, 2007.

[14] (2008). [Online]. Available: http://www.capsugel.com

[15] E. P. Furlani, Permanent Magnet and Electromechanical Devices. San Diego, CA: Academic Press, 2001 